\title{
Syndromic diarrhea
}

INSERM

\section{Source}

INSERM. (1999). Orphanet: an online rare disease and orphan drug data base. Syndromic diarrhea. ORPHA:84064

Syndromic diarrhea (SD), or tricho-hepato-enteric syndrome (THE), is a severe congenital enteropathy manifesting as intractable diarrhea in the first month of life with failure to thrive and associated with facial dysmorphism, hair abnormalities, and, in some cases, immune disorders and intrauterine growth restriction. 